\title{
Reabilitação Cardíaca no Enfarte Agudo do Miocárdio: Fatores Associados ao Sucesso
}

\author{
Carolina Pratas, ${ }^{1}$ Patrícia Coelho, ${ }^{2}$ José Aguila ${ }^{3}$
}

\begin{abstract}
RESUMO
Introdução: Os programas de reabilitação cardíaca têm como principal objetivo melhorar a tolerância ao exercício e aumentar a capacidade funcional do doente com patologia cardíaca, assim como educar e acompanhá-lo no controle dos seus fatores de risco cardiovasculares. Objetivo: Perceber quais os benefícios de um programa de reabilitação cardíaca em doentes que tenham sofrido enfarte agudo do miocárdio. Métodos: Os dados foram recolhidos por meio da consulta dos processos clínicos das pessoas que tiveram enfarte agudo do miocárdio entre 2015 e 2016, o que perfez um total de 20 indivíduos, dos quais 18 são do gênero masculino com idades entre os 46 e os 83 anos e 2 do gênero feminino, com 61 e 63 anos respetivamente. Foram ainda recolhidos os dados relativamente à frequência cardíaca, pressão arterial sistólica e diastólica, saturação de oxigênio e carga de trabalho antes e após a integração no programa de reabilitação. Resultados: Dos 20 indivíduos avaliados, verificou-se que existiu uma melhoria estatisticamente significativa entre os dois momentos de avaliação no índice de massa corporal com uma diminuição média de $1,73 \mathrm{Kg} / \mathrm{m}^{2}(p=0,023)$, uma diminuição média de $4,64 \mathrm{~cm}$ no perímetro abdominal $(p<0,001)$ e um aumento de $29 \mathrm{~W}$ na carga de trabalho $(p<0,001)$. Apesar de não se verificar uma relação estatisticamente significativa nas restantes variáveis, os dados obtidos demonstram que existe uma melhoria dos valores médios estudados entre os dois momentos. Discussão/conclusão: verifica-se que os indivíduos com enfarte agudo do miocárdio ao integrarem um programa de reabilitação cardíaca têm benefícios adicionais e independentes do tratamento farmacológico.
\end{abstract}

Palavras-chave: Enfarte agudo do miocárdio (C14.280.647.500). Reabilitação cardíaca (EO2.760.169.063.500.185). Doenças cardiovasculares (C14).

\section{CARDIAC REHABILITATION IN ACUTE MYOCARDIAL INFARCTION: FACTORS ASSOCIATED WITH SUCCESS}

\section{ABSTRACT}

Introduction: Cardiac rehabilitation programs aim to improve exercise tolerance and increase the functional capacity of patients with cardiac disease, as well as to educate and accompany them in the control of their cardiovascular risk factors. Objective: To understand the benefits of a cardiac rehabilitation program in patients who have suffered acute myocardial infarction. Methods: The data were collected through the consultation of the clinical processes of individuals who had acute myocardial infarction between 2015 and 2016, which made a total of 20 individuals, including 18 males aged 46 to 83 years and 2 of the genus female, 61 and 63 years respectively. Data were also collected regarding heart rate, systolic and diastolic blood pressure, oxygen saturation and workload before and after integration into the rehabilitation program. Results: Of the 20 individuals evaluated, there was a statistically significant improvement between the two moments of evaluation in the body mass index with a mean decrease of $1.73 \mathrm{~kg} / \mathrm{m} 2(p=0.023)$, a mean decrease of $4,64 \mathrm{~cm}$ in the abdominal perimeter $(p<0.001)$ and an increase of $29 \mathrm{~W}$ in the workload $(p<0.001)$. Although no statistically significant relationship was found in the remaining variables, the data obtained demonstrate that there is an improvement in the mean values studied between the two moments studied. Discusion/Conclusions: It is verified that individuals with acute myocardial infarction when integrating a cardiac rehabilitation program have additional and independent benefits of pharmacological treatment.

Keywords: Myocardial infarction (C14.280.647.500). Cardiac Rehabilitation (EO2.760.169.063.500.185). Cardiovascular Diseases (C14).

\footnotetext{
${ }^{1}$ Autora correspondente. Patient Care Specialist Linde Healthcare. carolinascpratas@gmail.com

2 Sport, Health \& Exercise Unit (SHERU). PhD, Instituto Politécnico de Castelo Branco.

${ }^{3}$ Fisiocardio. Badajoz.
} 


\section{INTRODUÇÃO}

Segundo a Organização Mundial da Saúde (OMS), a síndrome coronária aguda é atualmente uma das principais causas de morte mundial, causando mais de 1,9 milhão de mortes na União Europeia, e a sua incidência poderá ser diminuída caso sejam estabelecidas medidas de prevenção adequadas (BERRY; CUNHA, 2010; FERREIRA; ABREU, 2009).

Em Portugal, as Doenças Cardiovasculares (DCVs) têm uma elevada taxa de mortalidade e morbilidade, criando-se desta forma a imperiosa necessidade de programas de prevenção primária e secundária (MAGALHÃES, 2013). Estas patologias pelas graves consequências que interferem na qualidade de vida dos indivíduos que delas padecem, quer seja a nível físico, psicológico ou social (TAVARES, 2013), tornam incontornável a necessidade de complementar a terapêutica convencional (farmacológica) com a sua prevenção secundária, nomeadamente recorrendo aos Programas de Reabilitação Cardíaca (PRCs) (BERRY; CUNHA, 2010).

Apesar de a expressão qualidade de vida ser um conceito bastante utilizado por todos nós, a sua definição não se torna fácil. Segundo a OMS, esta representa a "perceção do indivíduo acerca da sua posição na vida, no contexto da sua cultura e sistema de valores, relativa aos seus objetivo, êxitos e padrões" (BETTENCOURT et al., 2005, p. 687-696).

Os PRCs foram criados com o intuito de promoverem uma melhoria da qualidade de vida dos sujeitos, permitindo-lhes uma recuperação física precoce após Enfarte Agudo do Miocárdio (EAM) e ainda uma orientação na reintegração social e retomada da atividade profissional. Estes programas estão organizados em três fases distintas, possibilitando uma avaliação otimizada dos indivíduos, tendo como objetivo primordial a que a sua integração não leve a um maior risco para o doente, mas sim ao melhor benefício. As medidas de prevenção secundária recomendadas para os PRCs têm como objetivo informar o doente sobre a patologia, promover a educação sobre as formas de prevenção, nomeadamente o controle dos Fatores de Risco Cardiovasculares (FRCVs) e a prescrição de um plano de exercícios físicos (de acordo com a estratificação do Risco Cardiovascular (RCV) de cada um) para promover uma melhoria de capacidade funcional e qualidade de vida destes indivíduos (FERREIRA; ABREU, 2009; MENDES, 2009). Percebe-se pelo descrito na literatura que apesar de o tratamento farmacológico das DCVs se mostrar eficaz, uma combinação deste com um PRC poderá vir a diminuir em número significativo a mortalidade e a morbidade destes indivíduos e aumentando-lhes a qualidade de vida e um controle assegurado destes fatores de risco.

Atendendo ao fato de a doença cardiovascular ser a maior causa de mortalidade e morbidade em Portugal e no mundo, é pertinente perceber quais os benefícios dos PRCs em indivíduos após EAM.

\section{MÉTODO DO ESTUDO}

De modo a analisar os fatores de sucesso inerentes a um PRC, efetuou-se um estudo observacional transversal, com indivíduos com cardiopatia isquêmica que ingressaram num PRC na Clínica Fisiocardio, em Badajoz - Espanha. Esta clínica é integrada por uma equipe de reabilitação multidisciplinar cuja atividade incide majoritariamente na prática da fase ॥ dos Programas de Reabilitação Cardíaca. O seu protocolo avalia inicialmente o indivíduo numa primeira consulta, quando são escrutinadas as capacidades físicas atuais e as possibilidades de recuperação. Nesse sentido, realiza-se uma ergometria e uma avaliação da musculatura periférica; verifica-se se o indivíduo tem dificuldades de aprendizagem e é elaborado um plano de exercícios. Uma vez realizada a primeira consulta e constituído o plano de exercícios, é necessário verificar com que intensidade devem ser realizados os exercícios - assegurando assim o máximo benefício e o menor risco. A intensidade do exercício vai ser adaptada a cada indivíduo em razão da frequência cardíaca máxima (calculada pela prova ergométrica basal) e o consumo de oxigênio (O2), sendo desta forma estabelecida uma Frequência Cardíaca de Exercício (FCE). $O$ treino recomendado de forma transversal é sempre de caráter dinâmico, de pouca duração e intensidade, podendo aumentar progressivamente e se alternar com exercícios de caráter isométrico. A sessão de treino no PRC começa pelo aquecimento - 10 minutos a andar; posteriormente inicia-se o treino aeróbico: 60\%-70\% da Frequência Cardíaca (FC) máxima obtida em ergometria sem sintomas - 30 a 40 minutos (min); exercícios complementares como: musculação e abdominais -15 min e por fim o alongamento - 10 min, que tem como objetivo principal o relaxamento. De forma a minorar os perigos e aumentar a eficácia da supervisão por parte da equipe de reabilitação, os doentes devem estar monitorados com eletrocardiograma basal, pressão arterial, frequência cardíaca e saturação de $\mathrm{O}_{2}\left(\right.$ Sat $\left.\mathrm{O}_{2}\right)$.

Para a realização deste estudo foram definidos como critérios de inclusão indivíduos com EAM que tenham integrado o programa de reabilitação cardía- 
ca entre 2015 e 2016, tendo sido a recolha de dados obtida por meio da consulta dos processos clínicos de cada um deles.

As variáveis recolhidas para este estudo foram: o gênero, a idade, a altura, o peso, o IMC (Índice de Massa Corporal) $\left(\mathrm{Kg} / \mathrm{m}^{2}\right)$, perímetro abdominal; tabagismo; Hipertensão Arterial (HTA); valor do colesterol (miligrama/decilitro - $\mathrm{mg} / \mathrm{dl}$ ), valor de High Density Lipoproteins (HDL) (em $\mathrm{mg} / \mathrm{dl})$; valor de Low Density Lipoproteins (LDL) $(\mathrm{mg} / \mathrm{dl})$, valor de triglicerídios (em $\mathrm{mg} / \mathrm{dl}$ ); presença de Diabetes Mellitus (DM); número de vasos ocluídos quando do diagnóstico do EAM; terapêutica utilizada após o evento cardíaco; presença de arritmias; variáveis relacionadas com a prova de esforço, como: duração da prova de esforço (min); frequência cardíaca (em batimentos por minuto - bpm) pré e pós-PRC; frequência cardíaca no pico do esforço (bpm); Pressão Arterial Sistólica (PAS) e diastólica (PAD) (em milímetros de mercúrio - $\mathrm{mmHg}$ ) pré e pós PRC; Sat $\mathrm{O}_{2}$ (em \%) pré e pós-PRC; fração de ejeção (em percentagem - \%), carga de trabalho (em watts W) pré e pós PRC e duração do PRC (em meses).

Os dados recolhidos foram tratados estatisticamente recorrendo ao programa Statistical Product and Service Solutions (SPSS), ${ }^{\circledR}$ versão 24.0, para o Windows. ${ }^{\circledR}$ A análise estatística baseou-se num estudo descritivo que permite uma investigação para caracterização da amostra, possibilita saber médias, desvio padrão, máximos e mínimos. Para além desta análise oportuniza ainda a realização de testes de normalidade, que averiguam se a amostra encontra-se dentro da normalidade. Considerando o número de indivíduos estudados foi utilizado o teste de Shapiro-Wilk $(n<30)$ com $p$-value $>0,05$, verificando assim que a amostra em estudo encontra-se dentro da normalidade. Para variáveis contínuas de distribuição normal recorreu-se ao teste paramétrico T-Student para amostras emparelhadas e para variáveis contínuas de distribuição não normal o teste não paramétrico Wilcoxon para amostras emparelhadas.

Para o recolhimento de dados o projeto de investigação foi submetido à Comissão de Ética da Escola Superior de Saúde Dr. Lopes Dias - Portugal - que depois de aprovado emitiu um ofício para a clínica Fisiocardio, do qual o diretor clínico teve conhecimento, autorizando assim a coleta dos dados para o estudo que foram recolhidos sob a forma de um código de números, garantindo assim a total confidencialidade dos indivíduos. A equipe de investigação comprometeu-se ainda a respeitar os princípios da Declaração de Helsinque e declara não haver conflito de interesses.

\section{RESULTADOS}

A amostra é constituída por 20 indivíduos, dos quais 18 são do gênero masculino - 90\% - e 2 do gênero feminino $-10 \%$.

Os indivíduos do gênero masculino apresentam idades entre 46 e 83 anos, com a média de 58,72 anos $\pm 9,015$ anos. A altura dos sujeitos variou entre 1,84 centímetros $(\mathrm{cm})$ e $1,60 \mathrm{~cm}$, tendo uma média de 1,73 $\mathrm{cm}$. No que diz respeito ao peso estes apresentam um valor mínimo de 70 quilogramas $(\mathrm{kg})$ e máximo de 124 $\mathrm{kg}$ ' com o peso médio de $87,2 \mathrm{~kg}$. Quanto ao gênero feminino as idades encontradas nos dois indivíduos foram 61 anos e 63 anos, respectivamente, com uma altura média de $1,60 \mathrm{~cm}$, o máximo de $1,65 \mathrm{~cm}$ e mínimo de $1,56 \mathrm{~cm}$; relativamente ao peso apresentam um mínimo de $51 \mathrm{~kg}$ e máximo de $51,5 \mathrm{~kg}$, com o peso médio de $51,2 \mathrm{~kg}$.

Os indivíduos em estudo apresentaram um IMC pré-PRC compreendido entre $18,70 \mathrm{~kg} / \mathrm{m} 2$ e 40,60 $\mathrm{kg} / \mathrm{m}^{2}$, constituindo o IMC médio de $28,38 \pm 5,63 \mathrm{~kg} /$ $\mathrm{m}^{2}$. Quanto ao perímetro abdominal estes apresentaram um valor compreendido entre 131 e $74 \mathrm{~cm}$, com o valor médio de 105,21 $\pm 14,60 \mathrm{~cm}$.

\section{Parâmetros Cardiovasculares}

\section{Fatores de Risco}

Analisamos os fatores de risco cardiovascular e verificamos que o mais predominante foi a hipertensão arterial, com $65 \%$ dos indivíduos, seguido do tabagismo, com $35 \%$. Apurou-se ainda que a maioria dos indivíduos (45\%) têm o colesterol dentro dos limites desejáveis e que $45 \%$ deles não tinham diagnóstico de DM (Quadro 1).

Quadro 1 - Caracterização dos fatores de risco da amostra em estudo

\begin{tabular}{|c|c|c|c|}
\hline \multirow{2}{*}{} & \multicolumn{2}{c|}{ Amostra em estudo } \\
\cline { 3 - 4 } & Não & $\mathbf{n}$ & $\%$ \\
\hline \multirow{4}{*}{ Tabagismo } & Sim & 7 & $25 \%$ \\
\cline { 2 - 4 } & Ex-fumador & 5 & $35 \%$ \\
\cline { 2 - 4 } & NA/NR & 3 & $15 \%$ \\
\cline { 2 - 4 } & Não & 6 & $30 \%$ \\
\hline \multirow{3}{*}{\begin{tabular}{c} 
Hipertensão \\
\cline { 2 - 4 }
\end{tabular}} & Sim & 13 & $65 \%$ \\
\cline { 2 - 4 } & NA/NR & 1 & $5 \%$ \\
\hline
\end{tabular}




\begin{tabular}{|c|c|c|c|}
\hline Colesterol & Desejável & 9 & $45 \%$ \\
\cline { 2 - 4 } & Risco Aumentado & 4 & $20 \%$ \\
\cline { 2 - 4 } & Elevado & 0 & $0 \%$ \\
\cline { 2 - 4 } & NA/NR & 7 & $35 \%$ \\
\hline \multirow{2}{*}{$\begin{array}{c}\text { Diabetes } \\
\text { Mellitus }\end{array}$} & Não & 9 & $45 \%$ \\
\cline { 2 - 4 } & Sim & 4 & $20 \%$ \\
\cline { 2 - 4 } & NA/NR & 7 & $35 \%$ \\
\hline
\end{tabular}

Legenda: n (número total); \% (percentagem); NA/NR (não aplicável/não resposta)

Fonte: Dados recolhidos pelos autores do estudo.

\section{Programa de Reabilitação Cardíaca}

Analisou-se o tempo que os indivíduos integraram o PRC e verificou-se que em média estes apresentaram entre 1 e 15 meses, tempo relativamente baixo para a realização de um PCR fase II.

\section{Parâmetros pré e pós-programa de reabilitação cardíaca}

Os indivíduos em estudo apresentaram uma frequência cardíaca basal pré-PRC entre os $49 \mathrm{bpm}$

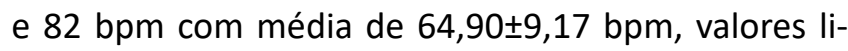
geiramente superiores aos encontrados no pós-PCR, que se registaram entre 53 e $72 \mathrm{bpm}$, com média de $65,78 \pm 6,06 \mathrm{bpm}$. No que diz respeito à fração de ejeção, os indivíduos apresentam um valor mínimo de $30 \%$ e máximo de $60 \%$, com uma média de $52,15 \pm 7,20$ $\%$, destacando-se que estes valores contemplam apenas os valores pré-PRC.

No estudo da pressão arterial verificou-se que a PAS apresentou valores pré-PRC entre $160 \mathrm{mmHg}$ e $100 \mathrm{mmHg}$, tendo uma média $123,25 \pm 19,14 \mathrm{mmHg}$ e a PAD valores entre $90 \mathrm{mmHg}$ e $60 \mathrm{mmHg}$, tendo como média $67,75 \pm 8,95 \mathrm{mmHg}$. Valores estes ligeiramente diferentes dos encontrados no pós-PRC, que se apresentaram para a PAS entre $200 \mathrm{mmHg}$ e $90 \mathrm{mmHg}$ e média de $117,89 \pm 24,39 \mathrm{mmHg}$ e para a PAD entre 70 $\mathrm{mmHg}$ e $60 \mathrm{mmHg}$, com uma média de $63,15 \pm 4,77$ $\mathrm{mmHg}$, mostrando assim haver uma melhoria dos valores médios da PA após um PRC.

Analisamos ainda a Sat $\mathrm{O} 2$, que apresentou valores entre $90 \%$ e $99 \%$, tendo como saturação média $95,60 \pm 2,32 \%$, valores semelhantes aos encontrados nos pós-PRC, que variaram entre os $90 \%$ e $98 \%$. Também neste caso se verificou uma média ligeiramente superior à da pré-PRC. Para a carga de trabalho pré-PRC, que variou entre os $24 \mathrm{~W}$ e $50 \mathrm{~W}$ com valor mé- dio $36 \pm 8 \mathrm{~W}$, esta aumentou no pós-PRC entre $5 \mathrm{~W}$ e $110 \mathrm{~W}$ e consequente o seu valor médio aumentou para $65 \pm 24,6 \mathrm{~W}$ (Quadro 2).

Quadro 2 - Parâmetros cardiovasculares pré e pós-Programa de Reabilitação Cardíaca

\begin{tabular}{|c|c|c|c|c|c|c|}
\hline \multirow{2}{*}{} & \multicolumn{5}{|c|}{ Indivíduos em estudo } \\
\cline { 2 - 7 } & \multicolumn{2}{|c|}{ Média \pm Desvio padrão } & \multicolumn{2}{|c|}{ Máximo } & \multicolumn{2}{c|}{ Mínimo } \\
\cline { 2 - 7 } & Pré & Pós & Pré & Pós & Pré & Pós \\
\hline FC (bpm) & $64,90 \pm 9,17$ & $65,78 \pm 6,06$ & 82 & 72 & 49 & 53 \\
\hline FE (\%) & $52,15 \pm 7,20$ & - & 60 & - & 30 & - \\
\hline $\begin{array}{c}\text { PAS } \\
\text { (mmHg) }\end{array}$ & $123,25 \pm 19,14$ & $117,89 \pm 24,39$ & 160 & 200 & 100 & 90 \\
\hline $\begin{array}{c}\text { PAD } \\
\text { (mmHg) }\end{array}$ & $67,75 \pm 8,95$ & $63,15 \pm 4,77$ & 90 & 70 & 60 & 60 \\
\hline $\begin{array}{c}\text { SatO2_pré } \\
\text { (\%) }\end{array}$ & $95,60 \pm 2,32$ & $96,21 \pm 1,78$ & 99 & 98 & 90 & 90 \\
\hline $\begin{array}{c}\text { Carga de } \\
\text { trabalho } \\
\text { pré (W) }\end{array}$ & $36 \pm 8$ & $65 \pm 24,6$ & 50 & 110 & 24 & 5 \\
\hline
\end{tabular}

Legenda: FC - frequência cardíaca; FE - fração de ejeção; PAS - pressão arterial sistólica; PAD - pressão arterial diastólica; SatO2 - saturação de oxigênio; W - carga de trabalho; bpm - batimento por minuto; \% - percentagem; $\mathrm{mmHg}$ - milímetros de mercúrio

Fonte: Dados recolhidos pelos autores do estudo.

\section{Efeitos do Programa de Reabilitação Cardíaca}

\section{Índice de massa corporal e perímetro abdominal}

Ao analisar o IMC nos dois momentos do PRC (primeira sessão versus última sessão) observou-se após o PRC uma variação estatisticamente significativa do IMC $(p=0,023)$, verificando-se que a maioria dos indivíduos diminuiu o valor do IMC em média $1,73 \mathrm{~kg} /$ $\mathrm{m}^{2}$ após o PRC, conforme é possível observar no Gráfico 1.

Gráfico 1 - Diferença das médias pré e pós-Programa de Reabilitação Cardíaca do IMC $(p=0,023)$

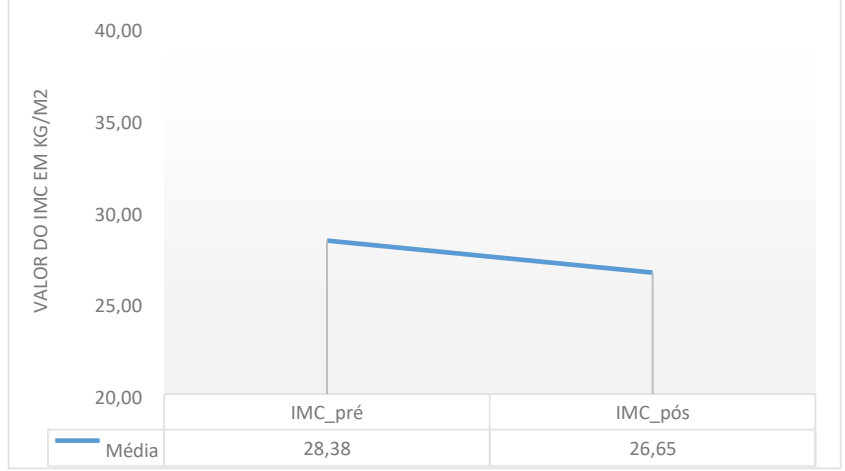

Legenda: IMC - índice de massa corporal; kg - quilograma; $\mathrm{m}^{2}$ - metro quadrado

Fonte: Dados recolhidos pelos autores do estudo. 
Fizemos a mesma análise para o perímetro abdominal e verificamos que também neste caso existe uma diferença estatisticamente significativa $(p<0,001)$ para esta variável, havendo uma diminuição em média de 4,64 cm do perímetro abdominal na maioria dos indivíduos que integraram o PRC (Gráfico 2).

Gráfico 2 - Diferença entre as médias pré e pós PRC de 4,64 cm programa de reabilitação cardíaca no PA $(p<0,001)$

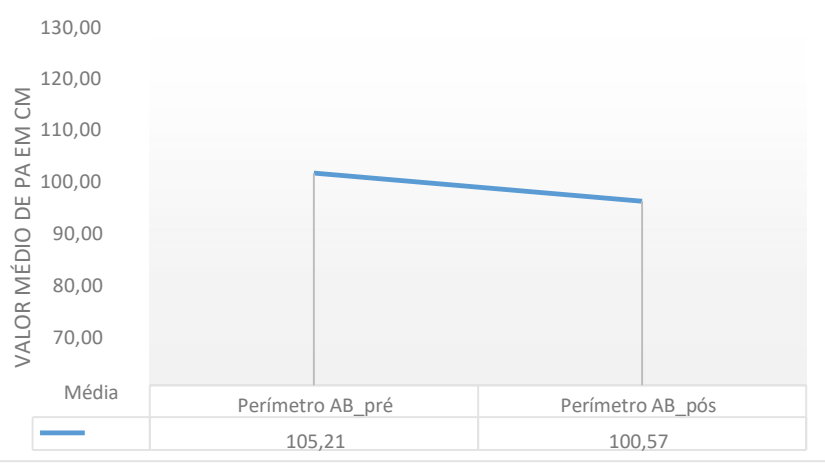

Legenda: PA - perímetro abdominal; $\mathrm{cm}$ - centímetros; $\mathrm{AB}$ - Abdominal Fonte: Dados recolhidos pelos autores do estudo.

\section{Frequência cardíaca}

Comparamos ainda os valores da FC antes e depois do PRC e após realizarmos o teste estatístico verificou-se não haver uma variação estatisticamente significativa $(p=0,479)$. Os valores médios encontrados mostraram que existe um aumento do valor médio de FC em quase metade da amostra.

Gráfico 3 - Pressão arterial sistólica em cada momento de avaliação, $(p=0,296)$

\section{Pressão arterial}

Para a análise da PAS e PAD antes e após o PRC utilizou-se o teste não paramétrico Wilcoxon. Constatou-se que antes do PRC os valores da PAS são maiores que no pós-PRC, no entanto quando comparados verifica-se não existirem diferenças estatisticamente significativas $(p=0,296)$ (Gráfico 3). Realça-se o fato de 11 dos 20 indivíduos terem diminuído o valor da PAS após o PRC. O mesmo verifica-se para a PAD; tal como podemos observar no Gráfico 4, este valor diminui entre o pré e o pós-PRC, mas também neste caso não se constatou diferenças estatisticamente significativas, no entanto o valor de $p$ aproxima-se mais do valor com significância estatística $(p=0,099)$ quando comparado com o valor de $p$ da PAS.

\section{Saturação de oxigênio}

Feita a análise dos valores da SatO2 no sangue antes e após o PRC, constatou-se que esta antes do PRC em média é menor que após o mesmo em $0,61 \%$. Para este aumento contribui o fato de 11 dos indivíduos estudados terem aumentado o valor da saturação após o PRC, no entanto quando comparados os valores entre os dois momentos verificou-se não existirem diferenças estatisticamente significativas $(p=0,413)$.

\section{Carga de trabalho}

Realizamos ainda testes para verificar qual a relação estatística entre as cargas de trabalho antes e após o PRC (Gráfico 5), o que revelou um aumento

Gráfico 4 - Pressão arterial diastólica em cada momento de avaliação, ( $p=0,099)$

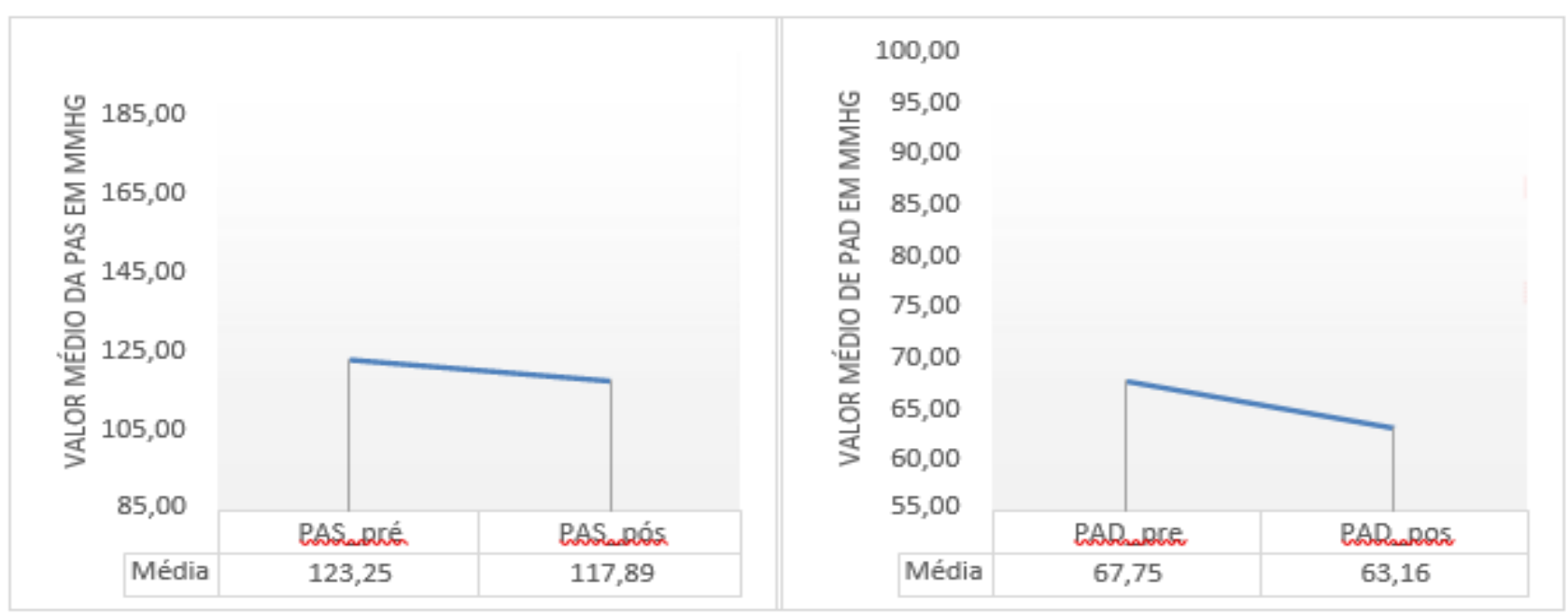

Legenda: PAS - pressão arterial sistólica; PAD - pressão arterial diastólica; $\mathrm{mmHg}$ - milímetros de mercúrio 
de carga significativo entre o início e o fim do programa. Esta relação mostra uma alta significância estatística entre os dois momentos $(p<0,001)$. Na análise desta variável percebemos que quase todos os indivíduos aumentaram a sua carga de trabalho durante o PRC.

Gráfico 5 - Diferença das médias entre o pré e pós-Programa de Reabilitação Cardíaca $(p<0,001)$

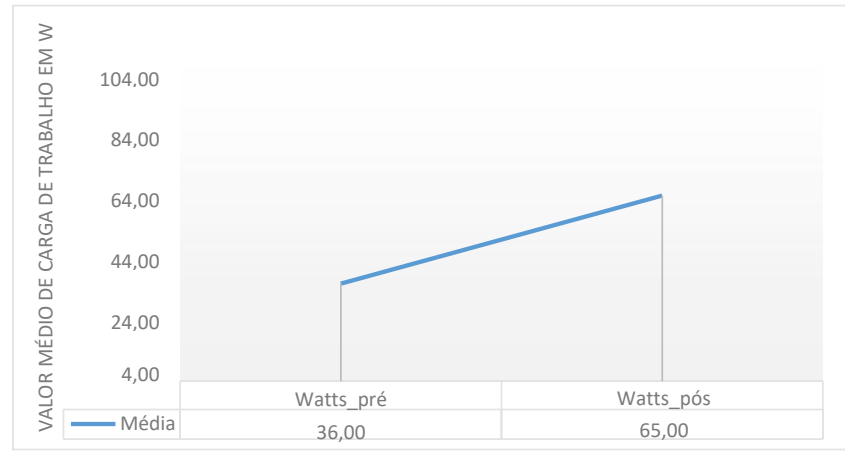

Legenda: W - carga de trabalho

Fonte: Dados recolhidos pelos autores do estudo.

Podemos assim constatar que existem benefícios estatisticamente significativos no perímetro abdominal, no IMC e na carga de trabalho e que apesar de na PAS, PAD e SatO ${ }_{2}$ não haver significância estatística os dados mostram-nos existir uma melhoria dos valores médios entre os dois momentos avaliados, tal como mostra o Quadro 3.

\section{DISCUSSÃO}

Os Programas de Reabilitação Cardíaca foram criados com o objetivo de promover uma recuperação acompanhada de uma forma rápida, proporcionando ao doente a sua reintegração social e profissional após um EAM (MENDES, 2009). Hoje os PRCs apresentam-se ainda como programas de prevenção secundária, instituindo assim o acompanhamento nutricional; observação da terapêutica farmacológica; prescrição de um programa de exercícios e sessões de ensino tanto para doentes quanto para familiares, de modo a auxiliá-los a viver com a doença (GIANNUZZI, 2003, 2008).

Em 2016, Nascimento et al. publicaram um estudo em que avaliaram 248 indivíduos antes e 6 meses após a participação num PRC, concluindo que estes obtiveram uma melhoria estatisticamente significativa no valor dos METS (equivalente metabólico) ao final dos 6 meses em PRC. No nosso estudo não foi possível ter acesso à prova de esforço dos indivíduos, uma vez que os exercícios aeróbio que estes realizavam durante as sessões eram em bicicleta com uma carga de trabalho adaptada a cada indivíduo. Constatamos que antes do PRC a carga de trabalho introduzida na bicicleta foi muito menor que aquela introduzida no seu final Esta diferença apresentou-se estatisticamente significativa, estando assim os nossos resultados de acordo com os resultados obtidos nos estudos de Nascimento et al. (2016) e Magalhães et al. (2013), que observaram uma melhoria no valor dos METS obtidos na prova de esforço, nos 12 meses de follow-up.

Em 2004, Caliani et al. publicaram um estudo com 133 indivíduos submetidos a um PRC e 40 indivíduos com terapêutica farmacológica convencional (grupo de controle). Nesse estudo observou-se uma diminuição do IMC a partir dos 3 meses em reabilitação cardíaca em comparação com o grupo de controle que não sofreu quaisquer alterações no IMC. Também o estudo de Magalhães et al. (2013) concluiu que o número de indivíduos cujo IMC era superior a $25 \mathrm{~kg} /$ $\mathrm{m}^{2}$ diminuiu o total de obesos de $19,9 \%$ para $16,4 \%$.

Quadro 3 - Benefícios da reabilitação cardíaca

\begin{tabular}{|c|c|c|c|}
\hline & Média \pm Desvio padrão pré & $\begin{array}{c}\text { Média } \pm \text { Desvio padrão } \\
\text { pós }\end{array}$ & $p$ \\
\hline IMC (kg/m²) & $28,38 \pm 5,63$ & $26,65 \pm 4,22$ & $0,023 *$ \\
\hline Perímetro Abdominal $(\mathrm{cm})$ & $105,21 \pm 14,60$ & $100,57 \pm 13,88$ & $<0,001^{*}$ \\
\hline FC (bpm) & $64,90 \pm 9,17$ & $65,78 \pm 6,06$ & $0,479 *$ \\
\hline PAS (mmHg) & $123,25 \pm 19,14$ & $117,89 \pm 24,39$ & $0,296 \#$ \\
\hline PAD (mmHg) & $67,75 \pm 8,95$ & $63,15 \pm 4,77$ & $0,099 \#$ \\
\hline Sat02 (\%) & $95,60 \pm 2,32$ & $96,21 \pm 1,78$ & $0,413^{*}$ \\
\hline Carga de trabalho (W) & $36 \pm 8$ & $65 \pm 24,6$ & $<0,001^{*}$ \\
\hline
\end{tabular}

Legenda: IMC - índice de massa corporal; FC - frequência cardíaca basal; PAS - pressão arterial sistólica; PAD - pressão arterial diastólica; SatO2 - saturação de oxigénio; \% - percentagem; $\mathrm{W}$ - carga de trabalho

* teste T-Student; \# teste de Wilcoxon

Fonte: Dados recolhidos pelos autores do estudo. 
Estes avaliaram ainda o perímetro abdominal, verificando-se uma redução estatisticamente significativa ao longo dos três momentos de avaliação. Também neste caso os resultados por nós encontrados revelam uma diminuição evidente do IMC e do perímetro abdominal antes e após o PRC, o que mostra que estes dois parâmetros analisados estão claramente associados ao sucesso e ao benefício que os doentes têm ao integrar um Programa de Reabilitação Cardíaca.

Outro parâmetro estudado foi a hipertensão arterial (HTA). A melhoria dos valores de pressão arterial está associada a exercícios submáximos, havendo estudos que comprovam que existe uma associação entre o nível de exercício físico e a melhoria dos valores de pressão arterial. Existem evidências clínicas dos benefícios do exercício para colmatar a HTA, reduzindo a sua gravidade, principalmente em indivíduos com HTA sistêmica. Fagard (2001) encontrou no seu estudo uma diminuição da PAS de $3,4 \mathrm{mmHg}$ e de $2,4 \mathrm{mmHg}$ na $\mathrm{PAD}$, observando-se que estes valores podem diminuir mais se o indivíduo for hipertenso (MONTERO, 2009; KODAMA, 2009; NASCIMENTO et al., 2016), o que está de acordo com o nosso estudo, que apesar de não mostrar diferenças estatisticamente significativas nem na PAS, nem na PAD, revelam uma diminuição clara dos valores entre os dois momentos avaliados.

Pela revisão da literatura percebemos que indivíduos com antecedentes de EAM, ao integrarem um Programa de Reabilitação Cardíaca, mesmo ocorrendo como prevenção secundária, este lhes traz benefícios no controle dos FRCVs, independentemente do tratamento farmacológico (BORTOLOTTO; CONSOLIM-COLOMBO, 2009; KRAEMER, 2013).

Sabe-se que a prática de exercício aumenta o débito cardíaco, levando assim a uma diminuição das resistências vasculares pulmonares, permitindo que a relação ventilação/perfusão seja mais eficaz. Este processo conduz a um aumento das trocas gasosas e assim a um aumento do aporte de oxigênio pelas células, logo a um aumento da saturação de oxigênio (MOREIRA, 2009). No nosso estudo, embora não se tenham verificado diferenças estatisticamente significativas entre o antes e após PRC, observou-se que dos 20 indivíduos, 11 tiveram um aumento da saturação de oxigênio ao final do programa.

Ao analisar o tempo que os indivíduos estiveram em PRC, verificamos que a média estava no limite inferior do tempo indicado pelas recomendações clínicas (FERREIRA; ABREU, 2009) para a fase II, que deve compreenderentre os 3 e os 6 meses, podendo-se ampliar para até um ano. É, no entanto, de salientar que foram encontrados benefícios em vários parâmetros cardiovasculares, mesmo que a média de tempo em PRC tenha sido baixa.

Embora conhecidos os seus benefícios, a RC continua a ser subutilizada. No final de 2007 Portugal ocupava o penúltimo lugar na Europa, com menos de $3 \%$ de indivíduos reabilitados (FERREIRA; ABREU, 2009). Muitas das razões para que ocorra esta subutilização está relacionada com a falta de diferenciação médica (falta de médicos especialistas na área de reabilitação cardíaca), de divulgação, motivação dos doentes e escassez na distribuição heterogênea dos centros de RC em Portugal e por último o déficit de coparticipação do Sistema Nacional de Saúde a esses sujeitos.

\section{CONCLUSÃO}

Apesar de o tempo em reabilitação cardíaca ter ocorrido no limite inferior do recomendado, os resultados encontrados mostram melhorias significativas no IMC, perímetro abdominal e no aumento da carga de trabalho.

Este estudo vem reforçar a relevância destes programas como uma forma de prevenção secundária, complementando a terapêutica convencional para o EAM, não só na melhoria dos FRCVs, mas também na melhoria da qualidade de vida destes indivíduos e eventual redução das internações dessas pessoas no pós-EAM.

\section{REFERÊNCIAS}

BERRY, J.; CUNHA, A. Avaliação dos efeitos da reabilitação cardíaca em indivíduos pós-infarto do miocárdio. Revista Brasileira Cardiologia, 23(2), p. 101-110, 2010.

BETTENCOURT, N. et al. Impacto da reabilitação cardíaca na qualidade-de-vida e sintomatologia depressiva após síndroma coronária aguda. Revista Portuguesa de Cardiologia, 25(5), p. 687-696, 2005.

BORTOLOTTO, L.; CONSOLIM-COLOMBO, F. Betabloqueadores adrenérgicos. Revista Brasileira de Hipertensão, 16(4), p. 215-220. 2009.

CALIANI, S. et al. Rehabilitación cardíaca postinfarto de miocardio en enfermos de bajo riesgo. Resultados de un programa de coordinación entre cardiología y atención primaria. Revista Espanhola Cardiologia, 57(1), p. 53-59, 2004.

FAGARD, R. H. Exercise characteristics and the blood pressure response to dynamic physical train-ing. Medicine \& Science in Sports \& Exercise, 33: S484-92, 2001. 
FERREIRA, D.; ABREU, D. Reabilitação Clínica: Realidade Nacional e Recomendações Clínicas. Grupos de Estudos fisiopatologia do Esforço e Reabilitação Cardíaca. Coordenação Nacional para as Doenças Cardiovasculares. Alto Comissariado da Saúde. S ISBN. Junho 2009.

GIANNUZZI, P. et al. Secondary prevention through cardiac rehabilitation: position paper of the Working Group on Cardiac Rehabilitation and Exercise Physiology of the European Society of Cardiology. European Heart Journal, 24(13), p. 1.273-1.278, jul. 2003.

GIANNUZZI, P. et al. Global secondary prevention strategies to limit event recurrence after myocardial infarction: results of the GOSPEL study, a multicenter, randomized controlled trial from the Italian Cardiac Rehabilitation Network. Archives Intern Medicine, 168(20), p. 2.194-2.204, Nov. 2008.

GRIMA, A. et al. Cardiología preventiva y rehabilitación cardiaca. Revista Espanhola Cardiologia, 64, Supl 1, p. 66-72, 2011.

KODAMA, S. et al. Cardiorespiratory fitness as a quantitative predictor of allcause mortality and cardiovascular events in healthy men and women: a meta-analysis. Jama, 301(19), p. 2.024-2.035, 2009.

KRAEMER, C. Resposta da frequência cardíaca de cardiopatas beta-bloqueados durante teste de força isocinético para grupo muscular da coxa. 2013. Dissertação (Mestrado Em Ciências do Desporto Especialização em Avaliação e Prescrição da Actividade Física) - Universidade de Trás-os-Montes e Alto Douro, Vila Real, 2013. p. 38-42.

MAGALHÃES, S. et al. Efeitos a longo prazo de um programa de reabilitação cardíaca no controlo dos fatores de risco cardiovasculares. Revista Portuguesa de Cardiologia, 32(3), p. 191-199, 2013.

MENDES, M. Reabilitação cardíaca em Portugal: a intervenção que falta! Saúde \& Tecnologia, 3, p. 5-9, 2009.

MONTERO, J. et al. Rehabilitación cardíaca en indivíduos com infarto de miocardio. Resultados tras 10 años de seguimiento. Revista Espanhola Cardiologia, 58, p. 1.181-1.187, 2005.

MONTERO, J. Rehabilitación cardíaca. Madrid: Acción Médica. Sociedad Española de Cardiología, 2009.

MOREIRA, L. A. Mecanismos de adaptação ao exercício físico. Porto . 2009.

NASCIMENTO, P. et al. Atividade física supervisionada meIhora a modulação autonómica de participantes de reabilitação cardíaca. Revista Portuguesa de Cardiologia, 1(35), p. 19-24, 2016.

TAVARES, N. et al. O efeito de um programa de exercício físico na qualidade de vida em indivíduos cardíacos. Revista Portuguesa de Saúde Pública, 31(1), p. 3-10, 2013. 